\title{
The association between caudal block and urethroplasty complications of distal tubularized incised plate repair: experience from a South China National Children's Medical Center
}

\author{
Jingqi Zhang", Shibo Zhu", Liyu Zhang, Wen Fu, Jinhua Hu, Zhao Zhang, Wei Jia \\ Department of Pediatric Urology, Provincial Key Laboratory of Research in Structure Birth Defect Disease, Guangzhou Women and Children's \\ Medical Center, Guangzhou Medical University, Guangzhou, China \\ Contributions: (I) Conception and design: S Zhu; (II) Administrative support: J Zhang; (III) Provision of study materials or patients: L Zhang; (IV) \\ Collection and assembly of data: J Zhang; (V) Data analysis and interpretation: J Zhang; (VI) Manuscript writing: All authors; (VII) Final approval of \\ manuscript: All authors. \\ "These authors contributed equally to this work. \\ Correspondence to: Wei Jia. Department of Pediatric Urology, Guangzhou Women and Children's Medical Center, Guangzhou Medical University, \\ Guangzhou, China. Email: jiawei198044@hotmail.com.
}

Background: The effect of caudal block $(\mathrm{CB})$ on the incidence of urethroplasty complications in hypospadias repair remains controversial. The evidence is conflicting, and some confounding bias issues need to be addressed. We sought to study a more homogenous group of distal hypospadias patients undergoing primary tubularized incised plate (TIP) repair by a senior pediatric urology surgeon in the past 2 years to examine the relationship between urethroplasty complications and the use of CB.

Methods: We reviewed our database to identify consecutive patients who had undergone hypospadias repairs by a senior director surgeon at our Center between January 2018 and November 2020. To be eligible to participate in the study, patients had to meet the following inclusion criteria: (I) have distal hypospadias; (II) have undergone a primary TIP repair; and (III) have attended follow-up appointments for a minimum period of 6 months. The primary outcome was the development of urethroplasty complications during the follow-up period. The principal variable of interest was whether or not CB was used perioperatively. The patients were categorized into a CB group (general anesthesia combined with CB) or a control group (general anesthesia only). Other potential risk factors were analyzed, including patient age at operation, patient weight, glans width, and the length of the urethral plate defect.

Results: Thirty (12.2\%) of the distal patients developed postoperative surgical complications. The postoperative surgical complication rates were similar between the different anesthesia groups. Weight, the length of the urethral plate length, and glans width did not contribute to the risk. Age was the only independent risk factor for postoperative surgical complications, and the complication rates increased in older patients.

Conclusions: Our data from consecutive TIP repairs in distal hypospadias patients indicated no association between the use of CB anesthesia and the postoperative urethroplasty complication rate. Patients who were older in age when they underwent surgery had a higher risk of complications.

Keywords: Hypospadias; tubularized incised plate repair (TIP repair); caudal block; fistulas

Submitted Apr 02, 2021. Accepted for publication May 10, 2021.

doi: $10.21037 /$ tau-21-355

View this article at: http://dx.doi.org/10.21037/tau-21-355 


\section{Introduction}

Hypospadias is one of the most common congenital anomalies in boys. From 1980-2010, the total prevalence of hypospadias was estimated to be 20.9 per 10,000 births, and in the last decades of the 20th century, the prevalence of hypospadias has increased (1). For glandular and penile shaft hypospadias repair, the procedure of choice is Tubularized urethroplasty with or without incision of the urethral plate (TIP). The urethroplasty complication rates, e.g., fistulas, meatal stenosis and urethral strictures are around $10 \%$ in TIP repairs; however, midshaft and proximal types have higher rates of complication $(2,3)$. Caudal epidural anesthesia is frequently used as an adjunct to general anesthesia in children during lower abdominal and genital surgeries, including hypospadias repair surgeries $(4,5)$. However, the effect of caudal block (CB) on the incidence of postoperative urethroplasty complications for hypospadias repair is controversial.

Several studies have reported that complication rates increase with the use of CB $(6,7)$. These researchers have theorized that the use of epidural CB might induce blood pooling and penile edema and result in delayed tissue healing (7). Conversely, 2 of the most recent large retrospective studies $(4,8)$ found no association between CB and the incidence of surgical complications in children who underwent hypospadias repair. There was also evidence that general anesthesia combined with CB elicits few or no hemodynamic alterations in children, especially in infants (9).

In addition to the conflicting results, some confounding bias issues also need to be addressed. First, some large retrospective studies have included cases that spanned more than 10 years or use more than 1 performing surgeon. Consequently, the matureness of surgical techniques and learning curve factors need to be considered and carefully interpreted. Second, the urethroplasty complication risk is associated with the severity of the disease, which is usually defined in relation to the original location of the urethra; the more proximal the starting point, the greater the risk that postoperative complications will develop. The selection of the regional anesthesia type for patients is based on anesthesiologists' decisions; however, observational studies have shown that a higher proportion of children with midshaft or proximal hypospadias receive CB compared to those with distal defects (8).

In this study, we sought to examine a more homogenous group of distal hypospadias patients who had undergone primary TIP repairs by a senior pediatric urology surgeon in the past 2 years to examine the relationship between $\mathrm{CB}$ and urethroplasty complications. We present the following article in accordance with the STROBE reporting checklist (available at http://dx.doi.org/10.21037/tau-21-355).

\section{Methods}

\section{Patient selection}

All procedures performed in this study involving human participants were in accordance with the Declaration of Helsinki (as revised in 2013). The study was reviewed and approved by the Ethics Committee of the Guangzhou Women and Children's Medical Center (No. 2020-657701). The participants' guardians provided written informed consent for the use of the participants' clinical data.

We reviewed our database to identify consecutive patients who had undergone hypospadias repairs by a senior director surgeon at our Center between January 2018 and November 2020. Patients' demographic, clinical, and surgical data were evaluated by a fellowship-trained pediatric urologist. To be eligible to participate in the study, patients had to meet the following inclusion criteria: (I) have distal hypospadias; (II) have undergone a primary TIP repair; and (III) have attended follow-up appointments for a minimum period of 6 months. Patients were excluded from the analysis if they had midshaft or proximal hypospadias, had underwent repairs using techniques other than TIP, had hypospadias combined with other urinary system malformations, had incomplete medical records, or had attended follow-up appointments for a period of less than 6 months.

The operation anesthesia strategy that each patient received was based on the random allocation of operation room. In 1 of 2 operation rooms designated for this surgeon's operations, general anesthesia combined with $\mathrm{CB}$ were administered, while in the other room, only general anesthesia was administered. For anesthesia induction, face mask sevoflurane was used. After securing intravenous access, propofol was intravenously administered in a single dose or repeated doses to maintain sedation. A consultant anesthesiologist then performed a caudal puncture with a 22 -gauge catheter by introducing in the caudal space; the patient was placed in the left lateral decubitus position. The mixed solution for the caudal injection was prepared with $0.9 \%$ saline and ropivacaine.

The surgical techniques and suture materials remained 
the same for all patients. All patients underwent TIP urethroplasty according to previously published techniques. Epinephrine was not used. A midline relaxing incision was made in the urethral plate, extending from the glans groove to the most proximal point of the plate and the midline skin closure was done with interrupted 7-0 epithelial polydioxanone (PDS) stitches. A compressive dressing was applied to the penis for 48 hours. A urethral catheter was left in place for 7 days. Patients received Cefaclor until catheter removal; anticholinergics were used as needed for bladder spasms.

Postoperatively, patients attended follow-up appointments at the Urology Outpatient Clinic of the Center or got phone call follow-up at 1, 3 and 6 months within first year and yearly thereafter to assess any postoperative urethroplasty complications.

\section{Measurements}

The primary outcome measure was the presence or absence of postoperative urethroplasty complications which included fistulas, glans dehiscence, and meatal stenosis. A fistula was observed as an abnormal connection between the reconstructed urethra and the original meatus skin and the tip of the glans, resulting in urine leakage (8). Meatal stenosis was defined if a 6-Fr sound would not traverse the meatus (10). Glans dehiscence was defined as a complete separation of the glans wings resulting in a coronal or more proximal meatus, or the complete separation of glans wings with an intervening bridge of skin (11). The principal variable of interest was whether or not $\mathrm{CB}$ was performed perioperatively. The patients were categorized into a $\mathrm{CB}$ group (general anesthesia combined with CB) or a control group (general anesthesia only). The other potential risk factors (covariates) analyzed in this study included patient age at operation [this continuous variable was measured in years and patients were classified into the following age groups: $<2$ years, $2-4$ years (not inclusive), and $\geq 4$ years]; patient weight (in kilograms); glans width (in centimeters), and the length of the urethral plate defect (in centimeters).

\section{Statistical analysis}

The statistical analyses were performed using SPSS 25 (IBM Corporation, Armonk, NY, USA) software. The descriptive statistics are presented as means and standard deviations
(SD) for the continuous variables and percentages for the nominal variables. Univariate analyses were conducted to test for a significant association between each risk factor and the primary outcome and to examine the distribution of covariates across the anesthetic groups. KolmogorovSmirnov tests were used to test for normal distribution of the data. Mann-Whitney $U$ tests were used for nonnormal distributed continuous variables. $T$-tests were used for normal distributed continuous variables. Categorical data were analyzed using Chi-square tests. If more than one factor was significant, these factors were included in a multivariate logistic regression analysis to estimate the adjusted odds ratios (ORs), and 95\% confidence intervals (CIs) were calculated for the ORs. All tests were 2-sided. A $\mathrm{P}<0.05$ was considered significant.

\section{Results}

\section{Cobort characteristics}

In total, 378 patients underwent TIP hypospadias repair by a senior urologists between Jan 2018-Nov 2020 at a single tertiary children's hospital. Six patients with incomplete medical records and 26 patients with less than 6 months follow-up were excluded, leaving 346 patients. Among these, 246 were distal type and were included for this analysis. Patients had a mean/median age of 2.61/2 years [SD 2.15; interquartile range (IQR) 2]. The mean/median weight was 13.32/12 kilograms (SD 13.32; IQR 4). The mean length of the urethral plate defect was $2.08 \mathrm{~cm}$ (SD 0.74). The mean size of glans was $1.35 \mathrm{~cm}$ (SD 0.21). Median follow-up time was 8 months. Overall, 130 patients $(52.85 \%)$ received a $\mathrm{CB}$. The results of the univariate analyses comparing the covariates between the anesthesia groups are set out in Table 1. There were no differences in relation to the covariates between the 2 groups.

Thirty (12.2\%) of the distal patients developed surgical complications. Complication rates were similar between the different anesthesia groups. Weight, urethral plate length defect, and glans size did not contribute to the risk. Age was the only independent risk factor for postoperative surgical complications, and the complication rates increased in older patients ( $\mathrm{RR}=1.18$; $95 \% \mathrm{CI}: 1.00-1.38$ ). The post-operation complication rates in patients who are younger than 2, aged from 2 to 4 and patients who are older than 4 were $7.1 \%, 11.6 \%$ and $22.6 \%$ respectively (Table 2). 
Table 1 Patients' demographic data between the different anesthesia groups

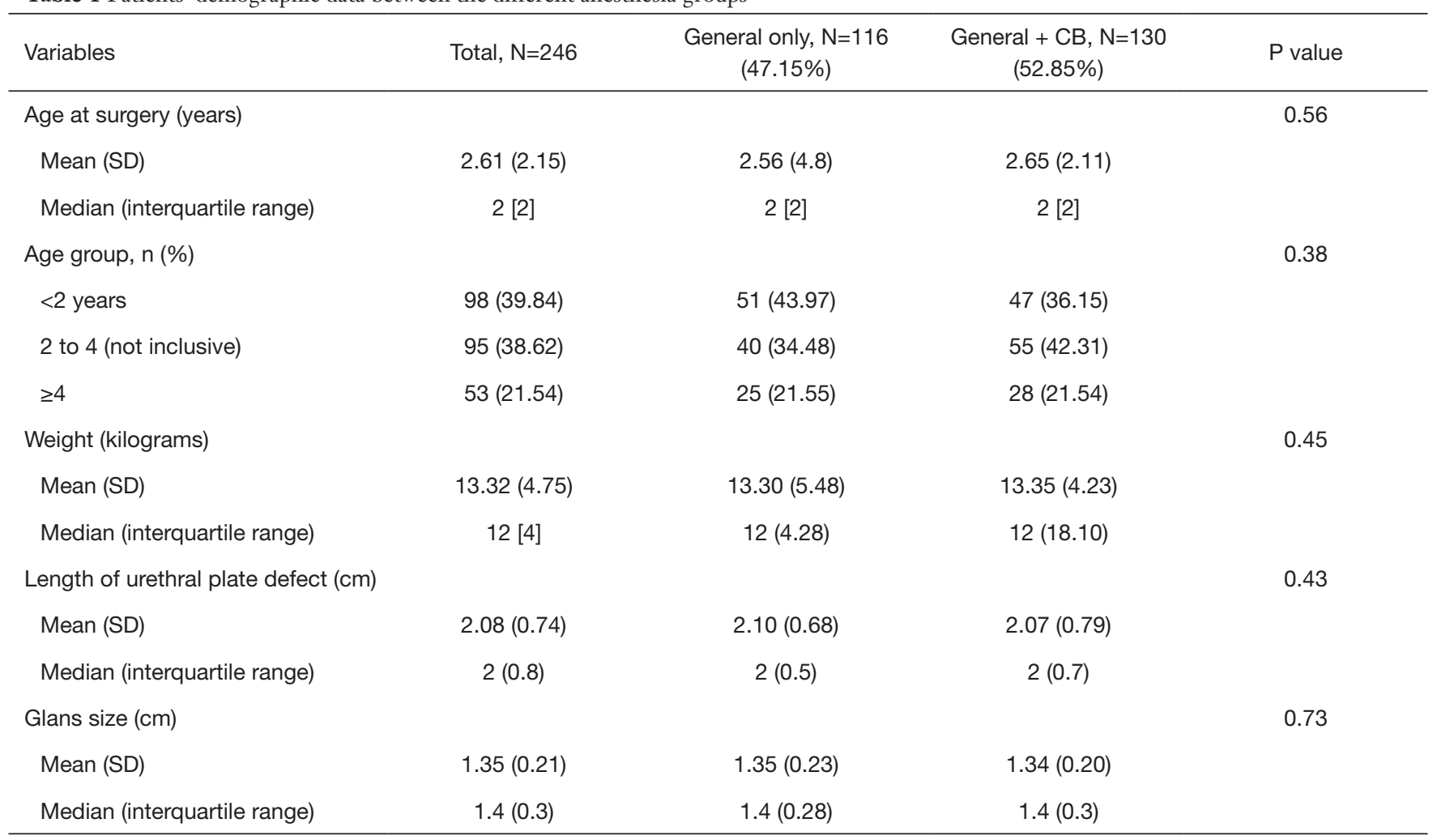

$\mathrm{CB}$, caudal block.

Table 2 Univariate and multivariable analysis of risk factors for urethroplasty complications in distal TIP repair patients

\begin{tabular}{|c|c|c|c|c|c|}
\hline Factors & $\begin{array}{l}\text { No complications, } \\
\qquad \mathrm{N}=216\end{array}$ & $\begin{array}{l}\text { Complications, } \\
\qquad \mathrm{N}=30\end{array}$ & $\frac{\text { Univariate }}{\mathrm{P} \text { value }}$ & \multicolumn{2}{|c|}{ Multivariate } \\
\hline Analgesia, n (\%) & & & & & 0.25 \\
\hline General only & 99 (85.3) & $17(14.7)$ & 0.27 & $1.58(0.73-3.45)$ & \\
\hline General + CB & $117(90.0)$ & $13(10.0)$ & & & \\
\hline Age group, n (\%) & & & 0.02 & & \\
\hline$<2$ years & $91(92.9)$ & $7(7.1)$ & & & \\
\hline 2 to 4 (not inclusive) & $84(88.4)$ & $11(11.6)$ & & & \\
\hline$\geq 4$ & $41(77.4)$ & $12(22.6)$ & & & \\
\hline Glans size (SD) & $1.34(4.47)$ & $1.36(0.24)$ & 0.44 & 0.65 (0.09-4.69) & 0.67 \\
\hline
\end{tabular}

TIP, tubularized incised plate; CB, caudal block, HR, hazard ratio. 


\section{Discussion}

TIP is a most commonly used procedure for distal and proximal hypospadias repair without severe chordee. Multiple risk factors have been identified as potential detriments to repair success, including the severity of the defect, the quality of the urethral plate, an older patient age, and the dexterity of the surgeon $(12,13)$.

We studied a more homogenous group of distal hypospadias patients who had undergone primary TIP repair by a senior pediatric urology surgeon in the past 2 years, and found no association between CB use and postoperative surgical complications. However, having an older age at the time of surgery was identified as a risk factor associated with higher complication rates.

The findings of our study about the effect of CB are consistent with those of Braga et al., who performed a retrospective analysis of patients, including TIP patients with distal hypospadias, from 2004-2015 ( $\mathrm{N}=405$ ). Braga et al. found similar complication rates for children who received CB and who did not (8). Zaidi et al. performed a retrospective nested case-control study on 114 distal 1 stage TIP repairs (between 1994 to 2013), and found no relationship between the use of $\mathrm{CB}$ and postoperative complications (14). However, in the above studies, the cases included those performed by 2 or more surgeons, and the study period spanned over 11 years. Thus, intersurgeon variability and surgeon case volume must be considered. Further, in a more recent meta-analysis of distal hypospadias, the author found that the type of regional block administered was not associated with postoperative complications (15).

Conversely, Saavedra-Belaunde et al. reviewed patients who underwent distal hypospadias repair by a single surgeon from 2008 to 2013, and observed that caudal anesthesia was associated with a higher risk of fistula occurrence (16). However, it should be noted that their study included patients who underwent tubularization of the urethral plate both with and without urethral plate incision, and was also limited by a relatively small sample size $(\mathrm{N}=192)$. Kim $e t a l$. and Taicher et al. both reported a higher risk of complications in patients who received caudal anesthesia compared to those who received another type of anesthesia. Both these studies included all types of hypospadias $(4,7)$. As we mentioned above, 1 observational study revealed that a higher proportion of children with midshaft/proximal hypospadias received CB compared to those with distal defects. However, the more proximal the starting point, the greater the risk that postoperative complications will develop. A randomized controlled study showed that CB was associated with a higher urethroplasty complication rate. In that study, of the 53 patients recruited, 19\% developed urethral fistulas and all received CB. However, it should be noted that in the research, hypospadias repair was performed by urologists or plastic surgeons, and the surgical techniques used in the CB group differed to those used in other groups.

The proposed mechanism for the increased complication risk of $\mathrm{CB}$ is that caudal vasodilation modifies the metabolic responses of tissues, leading to a decrease in the viscosity of blood and coagulability, and promoting the pooling of blood in penile tissue and penile engorgement $(6,16)$. CB has been shown to lead to a sympathetic block that provokes blood pooling in the denervated lower extremities in adults. However, in children and infants, caudal anesthesia, even when combined with general anesthesia, elicits few or no hemodynamic alterations, especially in patients younger than 8 years old (9). In infants, the use of CB was found to be associated with a significant increase in aortic blood flow and aortic stroke volume, and a decrease in lower body vascular resistance $(9,17)$. To explain this pediatric specificity, 2 hypotheses have been suggested. First, compared to adults, children have smaller lowerlimb blood volume, and second, their sympathetic nervous systems are "immature" (18). Children may have lower basal sympathetic tone than adults. A relative smaller lowerlimb blood volume in children may result in less blood pooling in the denervated lower extremities (19). Alizadeh et al. conducted a randomized clinical trial showing that CB could reduce the volume of blood loss during hypospadias repair. The mechanism might involve decreased arterial blood pressure due to sympathetic blockade and reduced venous tone and congestion in the surgical field (20). Further studies need to be conducted to gain a better understanding of the effects of caudal anesthesia on penile blood flow during and after hypospadias surgery.

Controversy remains as to whether age is a risk factor in the development of surgical complications. Hypospadias patients in developing countries, possibly due to family socioeconomic factors, access tertiary pediatric urology care at an older age. In our investigation, patients' age at operation was associated with a 1.58 -fold increase in the risk of urethroplasty complications. Our findings are supported by previous research. Notably, Yildiz et al. reviewed 307 boys with distal or midshaft penile hypospadias who underwent TIP repair, and found that patients in the 10-14 
age group had a significantly higher risk of developing complications than younger patients (12). Two studies in China showed that older children (children aged $>6$ ) who underwent hypospadias repair were at higher risk of urethrocutaneous fistulas after operation, while patients who were treated for hypospadias before 2-year-old had the least incidence of urethrocutaneous fistulas $(21,22)$. It has been suggested that children who undergo hypospadias repair at an age of older than 6 are more inclined to develop urethrocutaneous fistulas. Yildiz et al. reported a higher rate of urethrocutaneous fistulas in patients aged over 10 . There may be a number of reasons for the lower incidence of urethroplasty complications in younger patients. First, it might be that the healing ability of younger children is stronger than that of older children. Second, erections occur more frequently as age increases, and postoperative erections are considered a potential risk factor for postoperative bleeding and dehiscence, which affects postoperative complications, especially urethrocutaneous fistulas (22). Third, among adolescent or adult patients, skin appendages, such as hair follicles, are potential microbial reservoirs, which may lead to perioperative infections that affect the overall surgical success. In light of these investigations and our results, accessibility to healthcare services should be increased so that hypospadias can be treated earlier.

This study had several limitations that must be addressed. First, the retrospective nature of this study limited our ability to collect data on all the potential confounding factors. Second, we only included boys with the distal type of hypospadias defects. Conversely, the strengths of our study include the fact that our data consists of a large series of consecutive patients who were operated on by the same surgeon using the same operative technique during a time span of only 2 years. This allowed us to avoid any variations related to different surgeons using different operative techniques and changes in techniques due to time and learning curves.

\section{Conclusions}

Our data on consecutive TIP repairs in distal hypospadias patients indicated no association between the use of caudal regional anesthesia and postoperative complication rates. An older age at the time of operation was associated with a higher risk of complications. Thus, the importance of timely access to tertiary pediatric urology care for hypospadias patients, especially in developing countries, cannot be underestimated.

\section{Acknowledgments}

Funding: Guangzhou Institute of Pediatrics/ Guangzhou Women and Children's Medical Center (GWCMC2020-4-009) and Natural Science Foundation of Guangdong Province, China (Grant No.: 2019A1515011178).

\section{Footnote}

Reporting Checklist: The authors have completed the STROBE reporting checklist. Available at http://dx.doi. org/10.21037/tau-21-355

Data Sharing Statement: Available at http://dx.doi. org/10.21037/tau-21-355

Conflicts of Interest: All authors have completed the ICMJE uniform disclosure form (available at http://dx.doi. org/10.21037/tau-21-355). The authors have no conflicts of interest to declare.

Ethical Statement: The authors are accountable for all aspects of the work in ensuring that questions related to the accuracy or integrity of any part of the work are appropriately investigated and resolved. All procedures performed in this study involving human participants were in accordance with the Declaration of Helsinki (as revised in 2013). The study was reviewed and approved by the Ethics Committee of the Guangzhou Women and Children's Medical Center (No. 2020-657701). The participants' guardians provided written informed consent for the use of the participants' clinical data.

Open Access Statement: This is an Open Access article distributed in accordance with the Creative Commons Attribution-NonCommercial-NoDerivs 4.0 International License (CC BY-NC-ND 4.0), which permits the noncommercial replication and distribution of the article with the strict proviso that no changes or edits are made and the original work is properly cited (including links to both the formal publication through the relevant DOI and the license). See: https://creativecommons.org/licenses/by-nc$\mathrm{nd} / 4.0 \%$. 


\section{References}

1. Yu X, Nassar N, Mastroiacovo P, et al. Hypospadias Prevalence and Trends in International Birth Defect Surveillance Systems, 1980-2010. Eur Urol 2019;76:482-90.

2. Elbakry A. Management of urethrocutaneous fistula after hypospadias repair: 10 Years' experience. BJU Int 2001;88:590-5.

3. Shankar KR, Losty PD, Hopper M, et al. Outcome of hypospadias fistula repair. BJU Int 2002;89:103-5.

4. Taicher BM, Routh JC, Eck JB, et al. The association between caudal anesthesia and increased risk of postoperative surgical complications in boys undergoing hypospadias repair. Paediatr Anaesth 2017;27:688-94.

5. Wang HZ, Wang LY, Liang HH, et al. Effect of caudal ketamine on minimum local anesthetic concentration of ropivacaine in children: A prospective randomized trial. BMC Anesthesiol 2020;20:144.

6. Kundra P, Yuvaraj K, Agrawal K, et al. Surgical outcome in children undergoing hypospadias repair under caudal epidural vs penile block. Paediatr Anaesth 2012;22:707-12.

7. Kim MH, Im YJ, Kil HK, et al. Impact of caudal block on postoperative complications in children undergoing tubularised incised plate urethroplasty for hypospadias repair: a retrospective cohort study. Anaesthesia 2016;71:773-8.

8. Braga LH, Jegatheeswaran K, McGrath M, et al. Cause and Effect versus Confounding-Is There a True Association between Caudal Blocks and Tubularized Incised Plate Repair Complications? J Urol 2017;197:845-51.

9. Raux O, Rochette A, Morau E, et al. The Effects of Spread of Block and Adrenaline on Cardiac Output after Epidural Anesthesia in Young Children: A Randomized, DoubleBlind, Prospective Study. Anesth Analg 2004;98:948-55.

10. Bush NC, Holzer M, Zhang S, et al. Age does not impact risk for urethroplasty complications after tubularized incised plate repair of hypospadias in prepubertal boys. J Pediatr Urol 2013;9:252-6.

11. van der Toorn F, de Jong TPVM, de Gier RPE, et al. Introducing the HOPE (Hypospadias Objective Penile Evaluation)-score: A validation study of an objective scoring system for evaluating cosmetic appearance in hypospadias patients. J Pediatr Urol 2013;9:1006-16.

12. Yildiz T, Tahtali IN, Ates DC, et al. Age of patient is a risk factor for urethrocutaneous fistula in hypospadias surgery. J Pediatr Urol 2013;9:900-3.
13. Ru W, Shen J, Tang D, et al. Width proportion of the urethral plate to the glans can serve as an appraisal index of the urethral plate in hypospadias repair. Int J Urol 2018;25:649-53.

14. Zaidi RH, Casanova NF, Haydar B, et al. Urethrocutaneous fistula following hypospadias repair: Regional anesthesia and other factors. Paediatr Anaesth 2015;25:1144-50.

15. Zhu C, Wei R, Tong Y, et al. Analgesic efficacy and impact of caudal block on surgical complications of hypospadias repair: a systematic review and meta-analysis. Reg Anesth Pain Med 2019;44:259-67.

16. Saavedra-Belaunde JA, Soto-Aviles O, Jorge J, et al. Can regional anesthesia have an effect on surgical outcomes in patients undergoing distal hypospadia surgery? J Pediatr Urol 2017;13:45.e1-45.e4.

17. Monsel A, Salvat-Toussaint A, Durand P, et al. The Transesophageal Doppler and Hemodynamic Effects of Epidural Anesthesia in Infants Anesthetized with Sevoflurane and Sufentanil. Anesth Analg 2007;105:46-50.

18. Murat I, Delleur MM, Esteve C, et al. Continuous extradural anaesthesia in children. Br J Anaesth 1987;59:1441-50.

19. Deng $M$, Wang $X$, Wang L, et al. The hemodynamic effects of newborn caudal anesthesia assessed by transthoracic echocardiography: A randomized, doubleblind, controlled study. Paediatr Anaesth 2008;18:1075-81.

20. Alizadeh F, Heydari SM, Nejadgashti R. Effectiveness of caudal epidural block on interaoperative blood loss during hypospadias repair: A randomized clinical trial. J Pediatr Urol 2018;14:420.e1-420.e5.

21. Sheng X, Xu D, Wu Y, et al. The risk factors of Urethrocutaneous fistula after hypospadias surgery in the youth population. BMC Urol 2018;18:64.

22. Huang LQ, Ge Z, Tian J, et al. Retrospective analysis of individual risk factors for urethrocutaneous fistula after onlay hypospadias repair in pediatric patients. Ital J Pediatr 2015;41:35.

(English Language Editor: L. Huleatt)

Cite this article as: Zhang J, Zhu S, Zhang L, Fu W, Hu J, Zhang $Z$, Jia $W$. The association between caudal block and urethroplasty complications of distal tubularized incised plate repair: experience from a South China National Children's Medical Center. Transl Androl Urol 2021;10(5):2084-2090. doi: 10.21037/tau-21-355 